\title{
Colossal Pressure-Induced Softening in Scandium Fluoride
}

\author{
Zhongsheng Wei®, ${ }^{1, \dagger}$ Lei Tan $\odot,{ }^{1, \dagger}$ Guanqun Cai $\odot,{ }^{1, \dagger}$ Anthony E. Phillips $\odot,{ }^{1}$ Ivan da Silva $\odot,{ }^{2}$ \\ Mark G. Kibble, ${ }^{2}$ and Martin T. Dove $\oplus^{3,4, *}$ \\ ${ }^{1}$ School of Physics and Astronomy, Queen Mary University of London, Mile End Road, London E1 4NS, United Kingdom \\ ${ }^{2}$ ISIS Neutron and Muon Facility, Rutherford Appleton Laboratory, Harwell Campus, Didcot, Oxfordshire OX11 0QX, United Kingdom \\ ${ }^{3}$ College of Computer Science, Sichuan University, Chengdu, Sichuan 610065, People's Republic of China \\ ${ }^{4}$ Department of Physics, School of Sciences, Wuhan University of Technology, 205 Luoshi Road, \\ Hongshan district, Wuhan, Hubei 430070, People's Republic of China
}

(Received 2 April 2020; accepted 2 June 2020; published 25 June 2020)

\begin{abstract}
The counterintuitive phenomenon of pressure-induced softening in materials is likely to be caused by the same dynamical behavior that produces negative thermal expansion. Through a combination of molecular dynamics simulation on an idealized model and neutron diffraction at variable temperature and pressure, we show the existence of extraordinary and unprecedented pressure-induced softening in the negative thermal expansion material scandium fluoride $\mathrm{ScF}_{3}$. The pressure derivative of the bulk modulus $B, B^{\prime}=$ $(\partial B / \partial P)_{P=0}$, reaches values as low as $-220 \pm 30$ at $50 \mathrm{~K}$, and is constant at -50 between 150 and $250 \mathrm{~K}$.
\end{abstract}

DOI: 10.1103/PhysRevLett.124.255502

Just over 20 years ago a seminal paper on negative thermal expansion in $\mathrm{ZrW}_{2} \mathrm{O}_{8}$ [1] marked the beginning of a new area in materials chemistry and physics that has led to the discovery of many materials whose phonon behavior gives rise to highly anomalous physical properties. Since then we have seen not only many more materials showing negative thermal expansion, but also increases in the size of expansivities [2-4]. For example, around ten years ago, record positive and negative expansivities were reported in the network material $\mathrm{Ag}_{3} \mathrm{Co}(\mathrm{CN})_{6}$ [5]. Other counterintuitive properties related to atomic structure include negative linear compressibility [6] and negative Poisson's ratio [7]. More recently we have identified a new negative structural property, pressure-induced softening, in which the material becomes softer (less stiff) under pressure [8-11]. In this Letter we report a new instance of this property with an extremely large, indeed unprecedented, negative size of the associated coefficient.

Negative thermal expansion (NTE) is counterintuitive because any individual chemical bond always shows positive thermal expansion: it is harder to push two atoms together from the equilibrium distance than it is to pull them apart. Experimentally, positive thermal expansion of individual bonds has been directly observed in a number of materials, including the title material $\mathrm{ScF}_{3}$ [12-18]. The asymmetry in the distance dependence of the bond potential

Published by the American Physical Society under the terms of the Creative Commons Attribution 4.0 International license. Further distribution of this work must maintain attribution to the author(s) and the published article's title, journal citation, and DOI. means that at high temperature the vibration of the bond involves the bond stretching more than compressing, leading to an increase in the average separation, as illustrated in Fig. 1(a). This positive thermal expansion of the chemical bond usually leads to positive thermal expansion of the whole crystal. One common reason for negative thermal expansion of a crystal is called the "tension effect," which is illustrated in Fig. 1(b). Transverse motion of an atom strongly bonded to two neighbors on opposite sides will pull their mean positions inward, thereby reducing the average separation. This effect increases with temperature, leading to a negative expansivity, even with positive expansivities of the bonds. For a material such as the subject of this Letter, $\mathrm{ScF}_{3}$, the transverse atomic motions associated with the tension effect are seen in the atomic displacement parameters, as shown in Fig. 1(c). We recently quantified this using neutron scattering methods [18].

Pressure-induced softening is also counterintuitive, for similar reasons. The asymmetry of the potential energy of the chemical bond means that the more a bond is compressed, the harder it is to compress further, and the more the bond is stretched, the easier it is to stretch it further. Thus we expect any material to become stiffer as it is compressed. An familiar example is the snowball: it is soft when first formed, but then compression in the hand turns it into a hard sphere. However, in 1998 it was observed that amorphous silica shows the opposite behavior over the pressure range 0-1.5 GPa [19]. It is as if the snowball gets softer the more we squash it. Resistance to compression is quantified by the bulk modulus, $B=-V \partial P / \partial V$; its pressure dependence, $B^{\prime}=\partial B / \partial P$, is our quantity of interest here. A negative value of $B^{\prime}$ indicates pressureinduced softening [8-11]. We previously reproduced the 


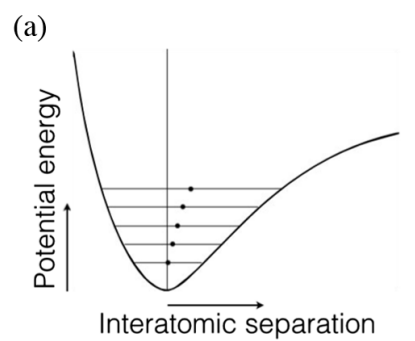

(b)

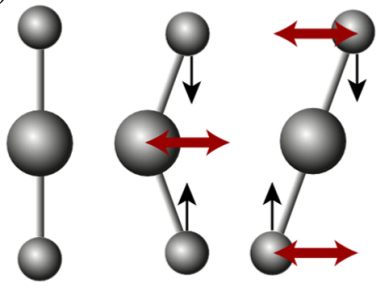

(c)

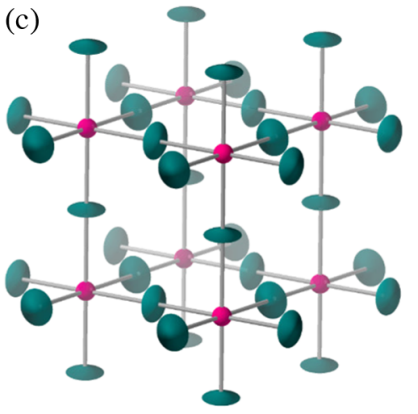

(d)

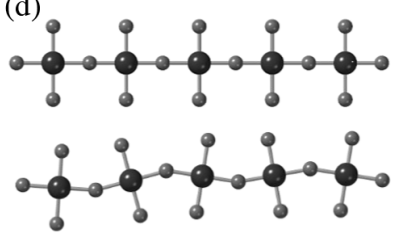

FIG. 1. (a) Representation of the potential energy of a bond between two atoms as a function of separation $r$ : the mean separation increases with temperature. (b) Illustration of the tension effect: transverse motions of an atom connected to two other atoms will pull the neighboring atoms together if the motions do not stretch the bonds. (c) Crystal structure of $\mathrm{ScF}_{3}$ showing transverse motions of the $\mathrm{F}$ atoms by representing the atoms as ellipsoids whose major axes reflect the thermal motion. (d) Top: A perfect alignment of atomic octahedra as in $\mathrm{ScF}_{3}$. In this arrangement compression (stretching) along the horizontal axis involves compression (stretching) of the bonds. Bottom: An elevated-temperature configuration where the thermal motion has rotated the octahedra. Compression or stretching now involves rotations of the bonds.

experimental negative value of $B^{\prime}$ in amorphous silica [19] using molecular dynamics simulations [20], and showed that the effect is strongly correlated with the same sort of structural fluctuations that give rise to negative thermal expansion.

Perhaps the best-known example of pressure-induced softening — which has attracted continued scientific interest precisely because of its rarity-is the minimum in the bulk modulus of cerium metal coinciding with the "volume collapse" at the transition from the $\gamma$ to the $\alpha$ phase [21-25]. This phase transition is driven by the electronic rather than the phonon structure, although the exact mechanism is still debated. The associated pressure-induced softening is characteristic of "pseudocritical" behavior above a structural phase transition [26]. In theory, such behavior can be described in terms of the specific heat critical exponent $\alpha$, with $B \sim\left(P_{c}-P\right)^{\alpha}$ and therefore $B^{\prime} \sim-\alpha B /\left(P_{c}-P\right)$, diverging to negative infinity at the critical pressure $P_{c}$. In practice, however, this cannot be used to achieve arbitrarily low $B^{\prime}$ : typically, a first-order phase transition occurs at a spinodal point well before this pressure [26]. Furthermore, $B^{\prime}$ values typically increase rapidly away from the critical point. At the critical point of $\mathrm{Ce}$, where the bulk modulus indeed drops to zero, $B^{\prime} \approx-58$, but this

increases rapidly as the temperature and pressure are changed, and in particular approaches zero at ambient pressure [25].

A simple explanation for a vibrational, rather than electronic, origin of pressure-induced softening is presented in Fig. 1(d), which shows two linear arrangements of octahedra. In the case of perfect alignment, compression or stretching of the system involves compressing or stretching the bonds between atoms. If these bonds are strong-which they usually are- the elastic modulus is large. At a raised temperature, thermal atomic motion involves crumpling of the arrangement. In this case, compression is made easier because it can now be achieved by further rotations of the bonds, requiring much less force in general than compressing bonds. This point has been discussed elsewhere in the context of the rigid unit mode model $[27,28]$, a set of ideas related to how the flexibility of the network of corner-shared structural polyhedra (such as $\mathrm{SiO}_{4}$ tetrahedra in silicates, $\mathrm{TiO}_{6}$ octahedra in perovskites, and $\mathrm{ScF}_{6}$ octahedra in the title material) impacts the nature of the lattice vibrations and thence on behavior such as phase transitions [28,29] and negative thermal expansion [30]. With large rotations, the projection of the longitudinal compressional force resolves more onto causing octahedral rotations than onto compression of individual bonds. Larger external pressure then causes even larger rotations, and the material becomes even softer. This is pressureinduced softening. Equivalently, we can consider stretching the crumpled chain: expansion first reduces the degree of rotation, until it reaches the point where further stretching of the system can only be accommodated by stretching the bonds, so that the system becomes elastically stiffer on stretching.

Pressure-induced softening has been observed in two materials that show negative thermal expansion, $\mathrm{ZrW}_{2} \mathrm{O}_{8}$ [31] and $\mathrm{Zn}(\mathrm{CN})_{2}$ [32]. We previously simulated pressureinduced softening in $\mathrm{Zn}(\mathrm{CN})_{2}$ by the molecular dynamics method [8], reproducing the experimental result. We also predicted a particular temperature dependence of $B^{\prime}$, which we subsequently confirmed experimentally [9]. Using an analytical model we showed that pressure-induced softening may be inevitable - rather than merely possible - in materials that show negative thermal expansion [10], and in a simulation of several zeolites we showed that this prediction is reasonable [11].

$\mathrm{ScF}_{3}$ has attracted a lot of interest for its negative thermal expansion [16,18,33-40]. The crystal structure is shown in Fig. 1(c): it is so irreducibly simple that it is effectively one of the few possible three-dimensional generalizations of the standard one-dimensional diatomic chain [41]. The cubic structure of $\mathrm{ScF}_{3}$ is exceptionally stable. In particular, unlike other $3 d$ transition metal trifluorides, at ambient pressure there is no sign of a phase transition to a rhombohedral structure down to $0.4 \mathrm{~K}$ [42], suggesting 


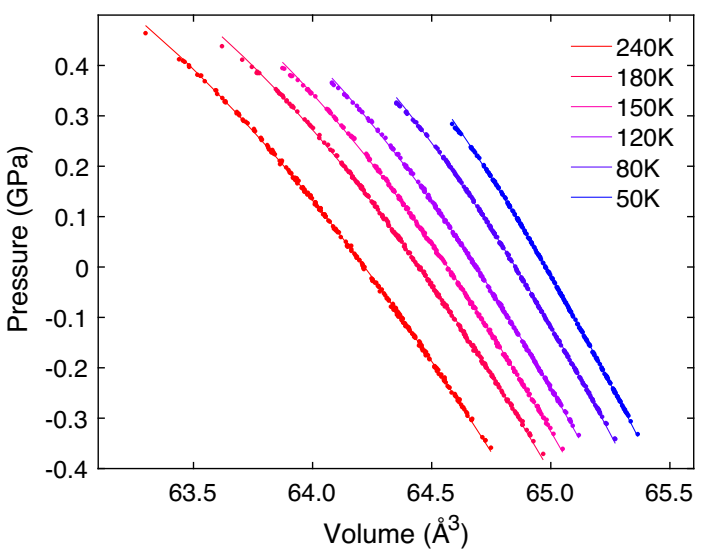

(a)

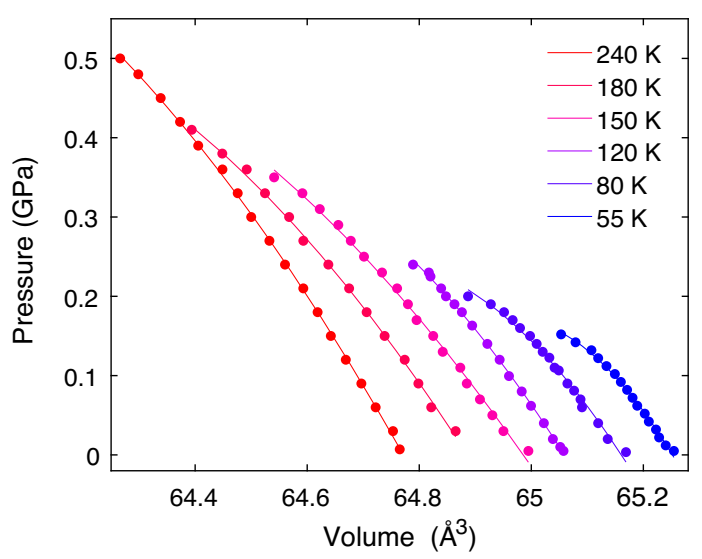

(b)

FIG. 2. $P(V)$ curves showing data from molecular dynamics simulation (a) and diffraction experiment (b) shown as filled circles, with the fitted third-order Birch-Murnaghan equation of state [Eq. (1)] shown as curves.

that the system is near a quantum phase transition [43]. On the other hand, the rhombohedral phase can be stabilized by an applied pressure of $0.7 \mathrm{GPa}$ at ambient temperature [44]. Because of both the anomalous thermal expansion and the proximity to a pressure-induced phase transition, one might expect this material to be a strong candidate for pressureinduced softening.

To explore the dynamics of the $\mathrm{ScF}_{3}$ structure, we developed a deliberately very simple atomistic model, with only a single bond-stretching interaction $(\mathrm{Sc}-\mathrm{F})$ and two bond-bending interactions ( $\mathrm{Sc}-\mathrm{F}-\mathrm{Sc}$ and $\mathrm{F}-\mathrm{Sc}-\mathrm{F}$ ); full details are given in the Supplemental Material [45]. The parameters were tuned by comparing the predictions with the results of $a b$ initio calculations [35]. Molecular dynamics simulations using this model showed negative thermal expansion, as expected (Fig. S1 in the Supplemental Material [45]).

We analyzed the pressure dependence of the atomic structure in the simulation over a wide range of temperatures, analyzing the results using the standard third-order Birch-Murnaghan equation of state:

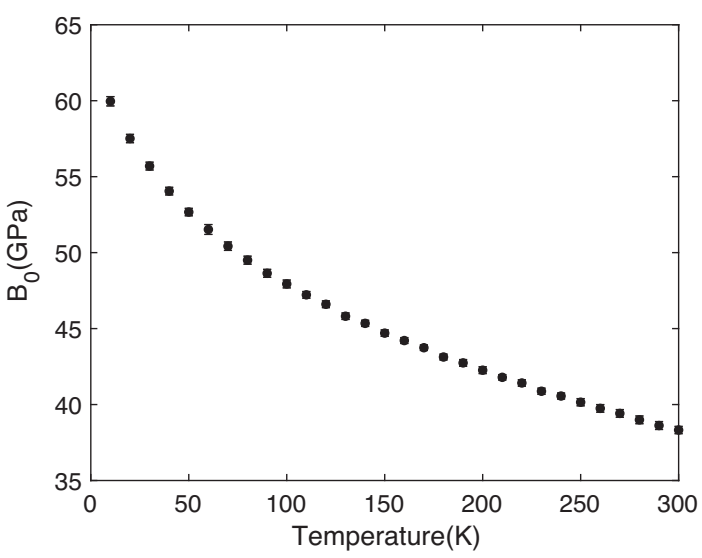

(a)

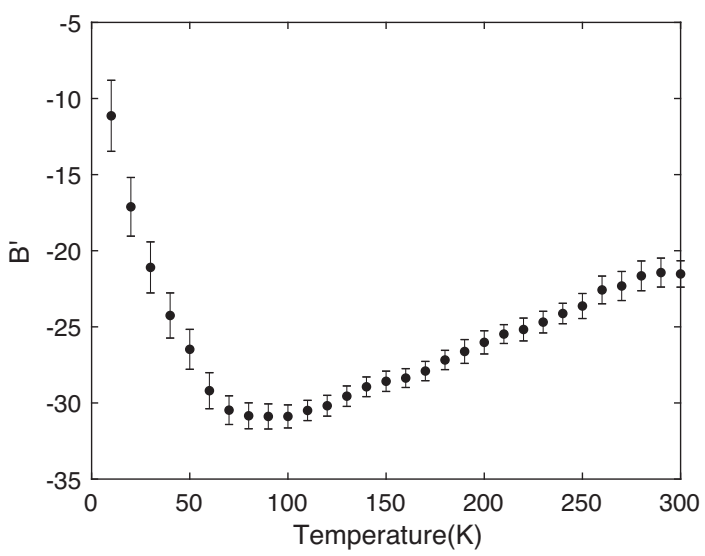

(b)

FIG. 3. Temperature dependence of $B_{0}$ (a) and $B^{\prime}$ (b) obtained from fitting the third-order Birch-Murnaghan equation of state to crystal volume obtained from molecular dynamics simulation of our idealized model for $\mathrm{ScF}_{3}$. The actual fitting is shown in Fig. 2(a).

$$
\begin{aligned}
P(V)= & \frac{3 B_{0}}{2}\left[\left(\frac{V_{0}}{V}\right)^{7 / 3}-\left(\frac{V_{0}}{V}\right)^{5 / 3}\right] \\
& \times\left\{1+\frac{3}{4}\left(B^{\prime}-4\right)\left[\left(\frac{V_{0}}{V}\right)^{2 / 3}-1\right]\right\},
\end{aligned}
$$

where $P$ and $V$ are the pressure and volume respectively, and $V_{0}$ and $B_{0}$ are the values of $V$ and $B$ at zero pressure. As an expansion of the second-order Birch-Murnaghan equation of state, the third-order equation reduces to the second-order form when $B^{\prime}=+4$, and many experimental systems actually have values of $B^{\prime}$ of roughly this size. Data for the functions $V(P)$ showing the fitted equation of state are shown in Fig. 2(a), and the fitted results for $B_{0}$ and $B^{\prime}$ are shown in Fig. 3. The temperature dependence of $B^{\prime}$ is very similar to that seen in $\mathrm{Zn}(\mathrm{CN})_{2}$ and zeolites [9,11,57], with a rapid change in the value of $B^{\prime}$ on heating from zero temperature, until reaching a minimum value (the most negative value) and then a slow change to less negative values on further heating [57]. What is astonishing is that our model for $\mathrm{ScF}_{3}$ predicts values of $B^{\prime}$ as extreme as -30 . 


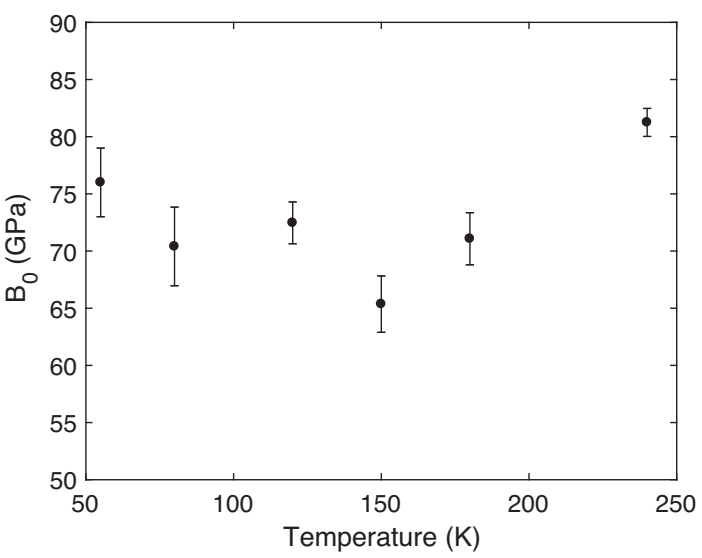

(a)

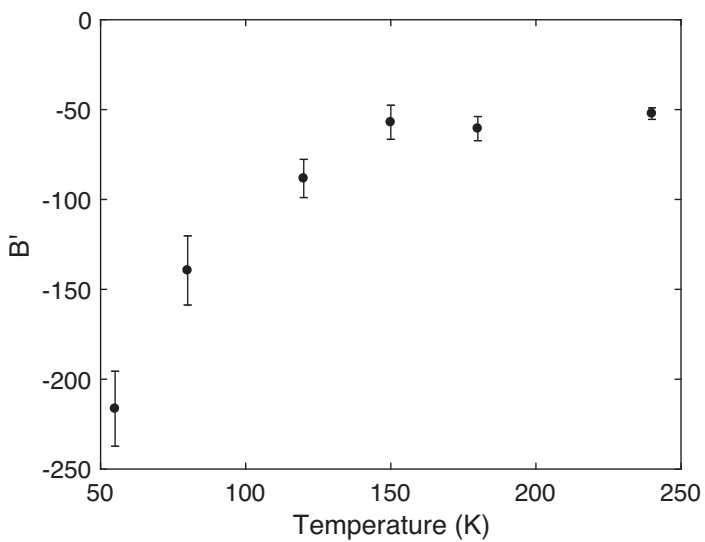

(b)

FIG. 4. Temperature dependence of $B_{0}$ (a) and $B^{\prime}$ (b) obtained from fitting the third-order Birch-Murnaghan equation of state to experimental data for the crystal volume as obtained from Rietveld analysis of diffraction data. The fitting is shown in Fig. 2(b)

As we noted above, negative values of $B^{\prime}$ are very rare; such a large negative value due to a phonon mechanism is unprecedented, and a comparable value is achieved by $\mathrm{Ce}$ only close to its critical point.

To follow up this prediction we performed neutron powder diffraction measurements of $\mathrm{ScF}_{3}$ under pressure for different temperatures on the GEM diffractometer at the ISIS spallation neutron facility. The diffraction patterns were fitted using the Rietveld method to obtain the lattice parameters and the atomic displacement parameters (all other aspects of the crystal structure are established by symmetry). All details of the experimental and analysis methods are given in the Supplemental Material, with a representative fit to the data shown in Fig. S2 [45].

The resultant experimental $P(V)$ for different temperatures were fitted by the third-order Birch-Murnaghan equation of state and shown in Fig. 2(b). The fitted values of $B_{0}$ and $B^{\prime}$ are shown in Fig. 4. The astonishing result is that the extraordinarily large negative values of $B^{\prime}$ predicted by simulation are not only reproduced but are exceeded in

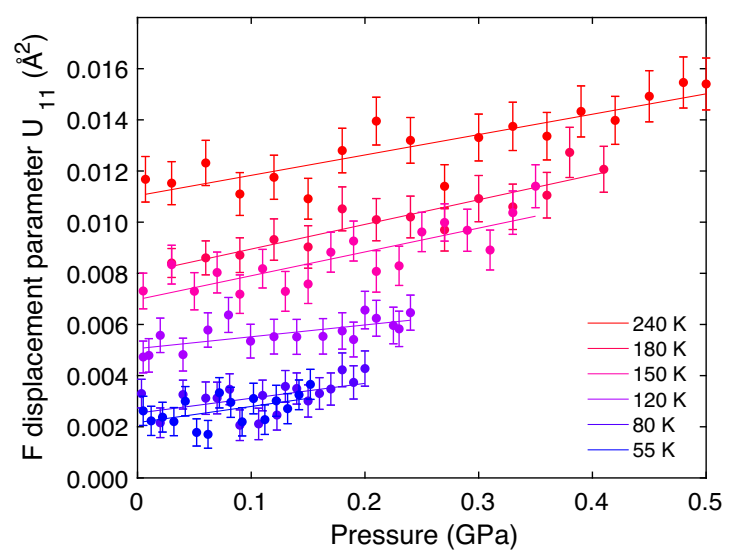

FIG. 5. Mean-squared transverse displacement of the F atoms obtained from refinement of the crystal structure.

the experimental results, with a greatest negative value of $B^{\prime}<-200$. We checked the robustness of this result by trimming the data range used in the fitting, and obtained results each time that are consistent within the estimated standard deviations. It might be argued that the third-order Birch-Murnaghan equation of state was not developed in anticipation of such large negative values of $B^{\prime}$, given that its derivation involves a Taylor expansion. However, the facts that the function fits the data to within the statistical accuracy, and that the fitting gives consistently large values of $B^{\prime}$ with relatively small estimated standard errors, are quantitative indications of the remarkable behavior seen here.

Figure 5 shows the fitted values of the fluorine transverse atomic displacement parameter-essentially the meansquare atomic displacement-as functions of pressure for different temperatures. This corresponds to the large transverse axis of the ellipsoids shown in the crystal structure, Fig. 1(c). At low pressure this quantity increases linearly with temperature as expected if the displacements are associated with thermal motions (Fig. S7 in the Supplemental Material [45]). Consistent with the ideas presented here, the data presented in Fig. 5 show that the mean-square amplitude of transverse motion of the fluorine atoms increases with pressure. On the other hand, in most materials the phonon frequencies increase under pressure due to force constants increasing, and higher frequencies lead to lower mean-square amplitudes of thermal motion. The behavior shown in Fig. 5 is consistent with enhanced rotations of the $\mathrm{Sc}-\mathrm{F}$ bonds on increasing pressure. By contrast, the mean-square amplitudes of the other motions (isotropic motion of the $\mathrm{Sc}$ atom, longitudinal motion of the $\mathrm{F}$ atom) show no pressure dependence (Fig. S8 in the Supplemental Material [45]). These results are reproduced in the results of the molecular simulations on the simple model (Fig. S5 in the Supplemental Material [45]).

To explain why the pressure-induced softening of $\mathrm{ScF}_{3}$ is so extreme, we consider our simple potential model. Recall that this has a stiff Sc-F bond, and two terms that control the flexing of the $\mathrm{F}-\mathrm{Sc}-\mathrm{F}$ right angle in the $\mathrm{ScF}_{6}$ 
octahedron and of the linear $\mathrm{Sc}-\mathrm{F}-\mathrm{Sc}$ angle. Our recent total scattering study of $\mathrm{ScF}_{6}$ [18] shows that there is considerable flexing of both these angles as compared with corresponding motions in other perovskites. The fact that NTE is largely absent in oxide perovskites suggests that this higher level of flexibility is essential for the mechanism of NTE, and by extension to the enhanced pressure-induced softening seen in $\mathrm{ScF}_{3}$. To confirm this, in separate simulations we increased the parameters governing each angle term to increase the forces opposing bending. We found that increasing either of these parameters leads to a clear reduction in NTE, indeed turning the thermal expansion into positive expansivity. The effect is strongest from the $\mathrm{F}-\mathrm{Sc}-\mathrm{F}$ angle term, where increasing the value of the force constant by a factor of only 3.3 is sufficient to drive the thermal expansivity to a positive value [18]. Our simulations show further that the same changes in the model parameters will reduce the negative size of $B^{\prime}$. This confirms that the enhanced pressure-induced softening in $\mathrm{ScF}_{3}$ is associated with the high degree of angular flexibility found in this material.

Such large negative values of $B^{\prime}$ as seen in this study for $\mathrm{ScF}_{3}$ are extraordinary: substantially greater than those for Ce metal, and greater still compared to other examples that arise from a vibrational rather than electronic mechanism. $\mathrm{Zn}(\mathrm{CN})_{2}$, for example, only has $B^{\prime}$ down to values of $-9.0 \pm$ 0.7 [9]. The values of $B^{\prime}$ seen in this study (Fig. 4) are so far outside the norm that it seems appropriate to recognize this by calling the effect "colossal pressure-induced softening" [58].

Because of the link we have demonstrated between anomalous thermodynamic properties, the same crystalengineering techniques used to design negative thermal expansion materials will be useful in discovering additional colossal pressure-induced softening materials. From a technological perspective, pressure-induced softening is particularly relevant to composite materials, where internal stress will be induced by differing thermal expansivities. By showing how extraordinarily large such effects can be, our results emphasize the importance of taking them into account when designing such composites. But we anticipate that colossal pressure-induced softening will also be a powerful tool that can be used to deliberately tune the thermodynamic behavior and physical properties of materials.

G. C. and L. T. are grateful for funding from the China Scholarship Council and Queen Mary University of London. Neutron beam time was provided by the ISIS Neutron and Muon Facility, operated under the auspices of STFC under Project No. RB1820458. Simulations were performed on the HPC Midlands Plus tier-2 system supported by EPSRC (EP/ P020232/1) (MTD coinvestigator).

*Corresponding author. martin.dove@icloud.com

${ }^{\dagger}$ These authors contributed equally to this work.
[1] T. A. Mary, J. S. O. Evans, T. Vogt, and A. W. Sleight, Negative thermal expansion from 0.3 to 1050 kelvin in $\mathrm{ZrW}_{2} \mathrm{O}_{8}$, Science 272, 90 (1996).

[2] K. Takenaka, Negative thermal expansion materials: technological key for control of thermal expansion, Sci. Technol. Adv. Mater. 13, 013001 (2012).

[3] C. P. Romao, K. J. Miller, C. A. Whitman, M. A. White, and B. A. Marinkovic, Negative thermal expansion (thermomiotic) materials, in Comprehensive Inorganic Chemistry II: From Elements to Applications (Elsevier, Oxford, 2013), pp. 127151.

[4] M. T. Dove and H. Fang, Negative thermal expansion and associated anomalous physical properties: review of the lattice dynamics theoretical foundation, Rep. Prog. Phys. 79, 066503 (2016).

[5] A. L. Goodwin, M. Calleja, M. J. Conterio, M. T. Dove, J. S. O. Evans, D. A. Keen, L. Peters, and M. G. Tucker, Colossal positive and negative thermal expansion in the framework material $\mathrm{Ag}_{3}\left[\mathrm{Co}(\mathrm{CN})_{6}\right]$, Science 319, 794 (2008).

[6] A. B. Cairns and A. L. Goodwin, Negative linear compressibility, Phys. Chem. Chem. Phys. 17, 20449 (2015).

[7] A. Alderson and K. L. Alderson, Auxetic materials, in Proceedings of the Institution of Mechanical Engineers, Part G (Journal of Aerospace Engineering) 221, 565 (2007).

[8] H. Fang, M. T. Dove, L. H. N. Rimmer, and A. J. Misquitta, Simulation study of pressure and temperature dependence of the negative thermal expansion in $\mathrm{Zn}(\mathrm{CN})_{2}$, Phys. Rev. B 88, 104306 (2013).

[9] H. Fang, A. E. Phillips, M. T. Dove, M. G. Tucker, and A. L. Goodwin, Temperature-dependent pressure-induced softening in $\mathrm{Zn}(\mathrm{CN})_{2}$, Phys. Rev. B 88, 144103 (2013).

[10] H. Fang, M. T. Dove, and A. E. Phillips, Common origin of negative thermal expansion and other exotic properties in ceramic and hybrid materials, Phys. Rev. B 89, 214103 (2014).

[11] H. Fang and M. T. Dove, Pressure-induced softening as a common feature of framework structures with negative thermal expansion, Phys. Rev. B 87, 214109 (2013).

[12] M. G. Tucker, M. T. Dove, and D. A. Keen, Direct measurement of the thermal expansion of the $\mathrm{Si}-\mathrm{O}$ bond by neutron total scattering, J. Phys. Condens. Matter 12, L425 (2000).

[13] Q. Hui, M. G. Tucker, M. T. Dove, S. A. Wells, and D. A. Keen, Total scattering and reverse Monte Carlo study of the $105 \mathrm{~K}$ displacive phase transition in strontium titanate, $\mathrm{J}$. Phys. Condens. Matter 17, S111 (2005).

[14] A. L. Goodwin, S. A. T. Redfern, M. T. Dove, D. A. Keen, and M. G. Tucker, Ferroelectric nanoscale domains and the $905 \mathrm{~K}$ phase transition in $\mathrm{SrSnO}_{3}$ : A neutron total-scattering study, Phys. Rev. B 76, 174114 (2007).

[15] M. J. Conterio, A. L. Goodwin, M. G. Tucker, D. A. Keen, M. T. Dove, L. Peters, and J. S. O. Evans, Local structure in $\mathrm{Ag}_{3}\left[\mathrm{Co}(\mathrm{CN})_{6}\right]$ : colossal thermal expansion, rigid unit modes and argentophilic interactions, J. Phys. Condens. Matter 20, 255225 (2008).

[16] L. Hu, J. Chen, A. Sanson, H. Wu, C. Guglieri Rodriguez, L. Olivi, Y. Ren, L. Fan, J. Deng, and X. Xing, New insights into the negative thermal expansion: Direct experimental 
evidence for the "guitar-string" effect in cubic $\mathrm{ScF}_{3}$, J. Am. Chem. Soc. 138, 8320 (2016).

[17] D. Wendt, E. Bozin, J. Neuefeind, K. Page, W. Ku, L. Wang, B. Fultz, A. V. Tkachenko, and I. A. Zaliznyak, Entropic elasticity and negative thermal expansion in a simple cubic crystal, Sci. Adv. 5, 2748 (2019).

[18] M. T. Dove, J. Du, Z Wei, D. A. Keen, M. G. Tucker, and A. E. Phillips, Quantitative understanding of negative thermal expansion in scandium trifluoride from neutron total scattering measurements, arXiv:1905.09250 [Phys. Rev. B (to be published)].

[19] O. B. Tsiok, V. V. Brazhkin, A. G. Lyapin, and L. G. Khvostantsev, Logarithmic Kinetics of the AmorphousAmorphous Transformations in $\mathrm{SiO}_{2}$ and $\mathrm{GeO}_{2}$ Glasses under High Pressure, Phys. Rev. Lett. 80, 999 (1998).

[20] A. M. Walker, L. A. Sullivan, K. Trachenko, R. P. Bruin, T. O. H. White, M. T. Dove, R. P. Tyer, I. T. Todorov, and S. A. Wells, The origin of the compressibility anomaly in amorphous silica: a molecular dynamics study, J. Phys. Condens. Matter 19, 275210 (2007).

[21] F. F. Voronov, V. A. Goncharova, and O. V. Stal'gorova, Elastic properties of cerium at pressures up to $84 \mathrm{kbar}$ and at a temperature of 293 K, Zh. Eksp. Teor. Fiz. 76, 1351 (1979) [JETP 49, 687 (1979)], http://www.jetp.ac.ru/cgi-bin/e/ index/e/49/4/p687?a=list.

[22] I.-K. Jeong, T. W. Darling, M. J. Graf, T. Proffen, R. H. Heffner, Y. Lee, T. Vogt, and J. D. Jorgensen, Role of the Lattice in the $\gamma \rightarrow \alpha$ Phase Transition of Ce: A HighPressure Neutron and X-Ray Diffraction Study, Phys. Rev. Lett. 92, 105702 (2004).

[23] M. J. Lipp, D. Jackson, H. Cynn, C. Aracne, W. J. Evans, and A. K. McMahan, Thermal Signatures of the Kondo Volume Collapse in Cerium, Phys. Rev. Lett. 101, 165703 (2008).

[24] F. Decremps, L. Belhadi, D. L. Farber, K. T. Moore, F. Occelli, M. Gauthier, A. Polian, D. Antonangeli, C. M. Aracne-Ruddle, and B. Amadon, Diffusionless $\gamma \rightleftharpoons \alpha$ Phase Transition in Polycrystalline and Single-Crystal Cerium, Phys. Rev. Lett. 106, 065701 (2011).

[25] M. J. Lipp, Z. Jenei, H. Cynn, Y. Kono, C. Park, C. KenneyBenson, and W.J. Evans, Anomalous elastic properties across the $\gamma$ to $\alpha$ volume collapse in cerium, Nat. Commun. 8, 1198 (2017).

[26] D. J. Bergman and B. I. Halperin, Critical behavior of an Ising model on a cubic compressible lattice, Phys. Rev. B 13, 2145 (1976).

[27] A. P. Giddy, M. T. Dove, G. S. Pawley, and V. Heine, The determination of rigid-unit modes as potential soft modes for displacive phase transitions in framework crystal structures, Acta Crystallogr. Sect. A 49, 697 (1993).

[28] K. D. Hammonds, M. T. Dove, A. P. Giddy, V. Heine, and B. Winkler, Rigid-unit phonon modes and structural phase transitions in framework silicates, Am. Mineral. 81, 1057 (1996).

[29] A. K. A. Pryde and M. T. Dove, On the sequence of phase transitions in tridymite, Phys. Chem. Miner. 26, 171 (1998).

[30] V. Heine, P. R. L. Welche, and M. T. Dove, Geometrical origin and theory of negative thermal expansion in framework structures, J. Am. Ceram. Soc. 82, 1793 (1999).
[31] F. R. Drymiotis, H. Ledbetter, J. B. Betts, T. Kimura, J. C. Lashley, A. Migliori, A. P. Ramirez, G. R. Kowach, and J. Van Duijn, Monocrystal Elastic Constants of the Negative-Thermal-Expansion Compound Zirconium Tungstate $\left(\mathrm{ZrW}_{2} \mathrm{O}_{8}\right)$, Phys. Rev. Lett. 93, 025502 (2004).

[32] K. W. Chapman and P. J. Chupas, Pressure enhancement of negative thermal expansion behavior and induced framework softening in zinc cyanide, J. Am. Chem. Soc. 129, 10090 (2007).

[33] B. P. Sobolev, The Rare Earth Trifluorides: Part 1: The High-Temperature Chemistry of the Rare Earth Trifluorides (Institut d'Estudis Catalans, Barcelona, 2000).

[34] B. K. Greve, K. L. Martin, P. L. Lee, P. J. Chupas, K. W. Chapman, and A.P. Wilkinson, Pronounced negative thermal expansion from a simple structure: $\mathrm{Cubic} \mathrm{ScF}_{3}$, J. Am. Chem. Soc. 132, 15496 (2010).

[35] C. W. Li, X. Tang, J. A. Muñoz, J. B. Keith, S. J. Tracy, D. L. Abernathy, and B. Fultz, Structural Relationship between Negative Thermal Expansion and Quartic Anharmonicity of Cubic $\mathrm{ScF}_{3}$, Phys. Rev. Lett. 107, 195504 (2011).

[36] C. R. Morelock, B. K. Greve, L. C. Gallington, K. W. Chapman, and A. P. Wilkinson, Negative thermal expansion and compressibility of $\mathrm{Sc}_{1-X} \mathrm{Y}_{X} \mathrm{~F}_{3}(x \leq 0.25)$, J. Appl. Phys. 114, 213501 (2013).

[37] L. Wang, C. Wang, Y. Sun, S. Deng, K. Shi, H. Lu, $\mathrm{P}$. $\mathrm{Hu}$, and $\mathrm{X}$. Zhang, First-Principles Study of $\mathrm{Sc}_{1-X} \mathrm{Ti}_{X} \mathrm{~F}_{3}(x \leq 0.375)$ : Negative Thermal Expansion, Phase Transition, and Compressibility, J. Am. Ceram. Soc. 98, 2852 (2015).

[38] L. Wang, C. Wang, Y. Sun, K. Shi, S. Deng, H. Lu, P. Hu, and $\mathrm{X}$. Zhang, Metal fluorides, a new family of negative thermal expansion materials, J. Mater. 1, 106 (2015).

[39] A. Corrales-Salazar, R. T. Brierley, P. B. Littlewood, and G. G. Guzmán-Verri, Landau theory and giant room-temperature barocaloric effect in $\mathrm{MF}_{3}$ metal trifluorides, Phys. Rev. Mater. 1, 053601 (2017).

[40] Y. Oba, T. Tadano, R. Akashi, and S. Tsuneyuki, Firstprinciples study of phonon anharmonicity and negative thermal expansion in $\mathrm{ScF}_{3}$, Phys. Rev. Mater. 3, 033601 (2019).

[41] The other is the simple rocksalt structure.

[42] C. P. Romao, C. R. Morelock, M. B. Johnson, J. W. Zwanziger, A. P. Wilkinson, and M. A. White, The heat capacities of thermomiotic $\mathrm{ScF}_{3}$ and $\mathrm{ScF}_{3}-\mathrm{YF}_{3}$ solid solutions, J. Mater. Sci. 50, 3409 (2015).

[43] S. U. Handunkanda, E. B. Curry, V. Voronov, A. H. Said, G. G. Guzmán-Verri, R. T. Brierley, P. B. Littlewood, and J. N. Hancock, Large isotropic negative thermal expansion above a structural quantum phase transition, Phys. Rev. B 92, 134101 (2015).

[44] K. S. Aleksandrov, V. N. Voronov, A. N. Vtyurin, S. V. Goryainov, N. G. Zamkova, V. I. Zinenko, and A. S. Krylov, Lattice dynamics and hydrostatic-pressure-induced phase transitions in $\mathrm{ScF}_{3}$, J. Exp. Theor. Phys. 94, 977 (2002).

[45] See Supplemental Material at http://link.aps.org/ supplemental/10.1103/PhysRevLett.124.255502 for more details on the crystal structure of $\mathrm{ScF}_{3}$; information about the molecular dynamics simulations with details of the method and implementation and some pertinent results not given in the main paper; and information about the neutron 
diffraction measurements with details of the method and analysis by Rietveld refinement, with some pertinent results not given in the main paper. The Supplemental Information contains Figs. S1-S8, and includes Refs. [46-56].

[46] J. D. Gale and A. L. Rohl, The general utility lattice program (GULP), Mol. Simul. 29, 291 (2003).

[47] J. D. Gale, GULP: A computer program for the symmetryadapted simulation of solids, J. Chem. Soc., Faraday Trans. 93, 629 (1997).

[48] L. H. N. Rimmer and M. T. Dove, Simulation study of negative thermal expansion in yttrium tungstate $\mathrm{Y}_{2} \mathrm{~W}_{3} \mathrm{O}_{12}$, J. Phys. Condens. Matter 27, 185401 (2015).

[49] I. T. Todorov, W. Smith, K. Trachenko, and M. T. Dove, DL_POLY_3: new dimensions in molecular dynamics simulations via massive parallelism, J. Mater. Chem. 16, 1911 (2006).

[50] M. Parrinello and A. Rahman, Crystal Structure and Pair Potentials: A Molecular-Dynamics Study, Phys. Rev. Lett. 45, 1196 (1980).

[51] S. Nosé, A unified formulation of the constant temperature molecular dynamics methods, J. Chem. Phys. 81, 511 (1984).
[52] W. G. Hoover, Canonical dynamics: Equilibrium phasespace distributions, Phys. Rev. A 31, 1695 (1985).

[53] S. Melchionna, G. Ciccotti, and B. Lee Holian, Hoover NPT dynamics for systems varying in shape and size, Mol. Phys. 78, 533 (2006).

[54] A.C. Hannon, Results on disordered materials from the GEneral Materials diffractometer, GEM, at ISIS, Nucl. Instrum. Methods Phys. Res., Sect. A 551, 88 (2005).

[55] O. Arnold et al., Mantid-Data analysis and visualization package for neutron scattering and SR experiments, Nucl. Instrum. Methods Phys. Res., Sect. A 764, 156 (2014).

[56] B. H. Toby and R. B. Von Dreele, GSAS-II: the genesis of a modern open-source all purpose crystallography software package, J. Appl. Crystallogr. 46, 544 (2013).

[57] H. Fang and M. T. Dove, A phenomenological expression to describe the temperature dependence of pressure-induced softening in negative thermal expansion materials, J. Phys. Condens. Matter 26, 115402 (2014).

[58] It has recently been reported that similar effects have been seen at room temperature in doped samples of $\mathrm{ScF}_{3}$ [Angus Wilkinson (private communication)]. 